\title{
Vigilância em Saúde em tempos de pandemia: análise dos planos de contingência dos estados do Nordeste
}

\section{Health surveillance in times of pandemic: analysis of contingency plans of Northeastern states}

\author{
Michael Ferreira Machadol,* iD \\ Túlio Romério Lopes Quirino",III iD \\ Carlos Dornels Freire de Souza' iD
}

\section{RESUMO}

Introdução: Apandemia da COVID-19 revelou uma série de desafios para os sistemas de saúde, exigindo tomadas de decisão rápidas e ações integradas para o seu contingenciamento. A Vigilância em Saúde é um dos elementos principais para a elaboração de respostas frente à pandemia. Objetivo: Analisar de que forma a Vigilância em Saúde tem sido pensada pelas estruturas de gestão estadual, no Nordeste brasileiro, considerando a proposição de medidas de contingenciamento para o enfrentamento à COVID-19. Método: Trata-se de estudo descritivo, de base documental, com caráter analítico-reflexivo, que tomou como objeto as publicações oficiais de secretarias estaduais de saúde dos estados nordestinos, produzidas a partir da elaboração de respostas governamentais à pandemia da COVID-19. 0 processo de análise, ancorado em pressupostos teórico-metodológicos da análise de conteúdo, considerou os seguintes componentes: (1) organização e estrutura formal; (2) objetivos definidos, gerais e específicos e (3) propostas definidas para a Vigilância em Saúde. Resultados: A análise levou à constituição de duas categorias: 1) aspectos políticos-gerenciais da Vigilância em Saúde e 2) articulações organizacionais da Vigilância em Saúde. A primeira discute a relação prevista entre as Vigilâncias em Saúde estaduais e municipais, com base na capacidade de institucionalização da gestão governamental. A segunda categoria foca a interface da Vigilância em Saúde com os demais setores da saúde e, na intersetorialidade. Conclusões: No contexto nordestino, desafios são colocados à organização de respostas da Vigilância em Saúde na gestão do enfrentamento à pandemia da COVID-19, destacando a capacidade de articulação intergovernamental de estados e municípios e a interface da Vigilância em Saúde com demais setores sanitários e sociais.

PALAVRAS-CHAVE: Vigilância em Saúde Pública; Pandemia; Gestão em Saúde

\section{ABSTRACT}

Introduction: The COVID-19 pandemic revealed a number of challenges for health systems, requiring rapid decision making and integrated actions for its contingency. Health Surveillance is one of the key elements in developing responses to the pandemic. Objective: To analyze how the Health Surveillance has been thought by the state management structures in the Northeast of Brazil, considering the proposal of contingency measures for the confrontation to COVID-19. Method: It is a descriptive study, documental-based, analytical-reflexive, which took as an object the official publications of state health secretariats of the northeastern states, produced from the elaboration of government responses to the COVID-19 pandemic. The analysis process, anchored in theoretical-methodological assumptions of content analysis, considered the following components: (1) formal organization and structure; (2) defined objectives, general and specific and; (3) defined proposals for Health Surveillance. Results: The analysis led to the constitution of two categories: 1) political-managerial aspects of Health Surveillance; 2) organizational articulations of Health Surveillance. The first category discusses the relationship between state and municipal Health Surveillance, based on the capacity of institutionalization of government management. The second category focuses on the 
interface of the Health Surveillance with other health sectors and, in intersectoriality. Conclusions: In the northeastern context, challenges are posed to the organization of Health Surveillance responses in the management of the COVID-19 pandemic response, highlighting the intergovernmental articulation capacity of states and municipalities and the Health Surveillance interface with other health and social sectors.

KEYWORDS: Public Health Surveillance; Pandemics; Health Management

\section{INTRODUÇÃO}

A pandemia da COVID-19 constitui uma unanimidade entre as preocupações contemporâneas da gestão dos sistemas de saúde em âmbito mundial. Desde o seu surgimento, ao final de 2019, como um surto epidêmico de rápida dispersão na região de Hubei, na China, esta nova síndrome respiratória se comportou com grau alarmante de disseminação entre todos os cantos do globo, atingindo a Europa e os Estados Unidos no mês de janeiro de 2020, e, em fevereiro, o Brasil ${ }^{1,2}$.

Normativas institucionais e resoluções editadas pela Organização Mundial da Saúde (OMS) e outras entidades sanitárias, além de sucessivos decretos e portarias publicados pelos governos federal, estaduais e municipais, têm demonstrado ao longo de sua permanência no território nacional, há aproximadamente quatro meses, seu grau crescente de importância à manutenção das condições de vida da sociedade brasileira, imperando reclassificações de seu grau de ameaça à integridade física e de saúde da população em geral ${ }^{1,3,4,5}$. 0 que levou a tomadas de decisão político-institucionais urgentes, como respostas-rápidas ao espalhamento da pandemia, envolvendo medidas de proteção individuais e coletivas, como a suspensão no funcionamento de serviços e a adoção de novos hábitos sanitários e comportamentais ${ }^{6}$.

Tal panorama, embora de origem sanitária, tem repercutido também no agravamento de problemáticas sociais de diversas ordens, ao tangenciar questões relacionadas às condições de habitação, alimentação, trabalho, renda, acesso a bens de consumo, entre outras. Logo, o desafio posto ultrapassa o setor saúde, fazendo emergir pautas que há muito carecem de atenção e discussão, catapultadas, em especial, pela crise econômica que acompanha a proliferação desse novo coronavírus (2019-nCoV) $)^{7,8}$.

É, no entanto, no âmbito da gestão dos sistemas de saúde que os desafios se colocam como imperativo, demandando de seus representantes e equipes habilidades técnicas e competências políticas para minorar os efeitos da pandemia em cada contexto loco-regional, tendo em vista que a ação patogênica do vírus tem revelado a sobrecarga nos sistemas de saúde por todo o mundo ${ }^{7,8}$. Assim, no Brasil, há que se destacar a difícil tarefa dos gestores da saúde, em seus diferentes níveis, no cumprimento da missão a eles impetrada desde a Constituição Federal de 1988, qual seja: a garantia do direito universal, integral e equânime da saúde a toda a população brasileira9.

A Constituição Brasileira assegura a saúde como um direito de todos e um dever do Estado ${ }^{10}$. A forma de executar essa orientação constitucional foi a implantação do Sistema Único de Saúde (SUS). Uma das características do SUS é a descentralização de ações e dos seus serviços, o que contribui para a qualificação do sistema de saúde, na medida em que aproxima os aspectos sociais, políticos e administrativos com as especificidades epidemiológicas, com vistas a superar as fragmentações das políticas e dos programas de saúde, por meio de uma rede regionalizada de ações ${ }^{11}$.

Dessa forma, desde a década de 1990, no Brasil, os municípios, com apoio dos estados e da união, tornaram-se os principais responsáveis pela gestão da rede de serviços de saúde e os gestores municipais assumiram o papel fundamental na execução das ações e serviços de saúde, atendendo as particularidades de cada região ${ }^{12}$.

Uma das ações descentralizadas no âmbito do SUS, instituídas por meio do processo de pactuação intergestores, foi a Vigilância em Saúde, compreendida como

um processo contínuo e sistemático de coleta, consolidação, análise e disseminação de dados sobre eventos relacionados à saúde, visando o planejamento e a implementação de medidas de saúde pública para a proteção da saúde da população, a prevenção e controle de riscos, agravos e doenças, bem como para a promoção da saúde ${ }^{13}$.

O uso efetivo e sistemático das informações da Vigilância em Saúde torna-se fundamental para que estados e municípios consigam definir prioridades, aperfeiçoem a distribuição de recursos e elaborem qualificadas estratégias de intervenção em saúde ${ }^{14}$. Logo, compreender o planejamento e o desenvolvimento de ações de Vigilância em Saúde consiste em exercício imprescindível aos distintos níveis de gestão do sistema de saúde, especialmente, em contextos de pandemia.

É a partir desta premissa que se desenvolve o presente artigo, o qual pretendeu analisar de que forma a Vigilância em Saúde tem sido pensada pelas estruturas de gestão estadual, no Nordeste brasileiro, considerando a proposição de medidas de contingenciamento para o enfrentamento à COVID-19 inscritas nos planos de contingência elaborados pelas suas equipes gestoras.

\section{MÉTODO}

Este artigo, de caráter analítico-reflexivo, utilizou como objeto de problematização as publicações oficiais de secretarias estaduais de saúde dos estados nordestinos, produzidas no âmbito da elaboração de respostas governamentais à pandemia da COVID-19. Origina-se, portanto, de um estudo descritivo de base documental, que buscou reconhecer de que maneira estes documentos apresentam direcionamentos à tomada de decisão em 
saúde, que possibilitem o enfrentamento à pandemia em seus territórios de abrangência.

Para o seu desenvolvimento, tomamos como referência a estratégia metodológica definida por Spink ${ }^{15}$ como trabalho de "catar documentos", a qual viabiliza a aproximação e análise de documentos de domínio público, alinhando-se às pesquisas que elegem o cotidiano como objeto de problematização ${ }^{16,17}$.

Para este autor ${ }^{15}$, os documentos de domínio público consistem em "produtos sociais tornados públicos", e de tal forma, estão "eticamente abertos para análise por pertencerem ao espaço público, por terem sido tornados públicos de uma forma que permitem a responsabilização" ${ }^{17}$. Assim, a análise de tais documentos é propícia, já que "podem refletir as transformações lentas em posições e posturas institucionais assumidas pelos aparelhos simbólicos que permeiam o dia-a-dia”, podendo revelar achados importantes para ponderar e debater os movimentos da sociedade, no tempo e espaço em que operam.

Neste trabalho, foram considerados para análise os planos de contingência elaborados pelos governos estaduais dos nove estados que compõem a Região Nordeste do Brasil, a qual concentra atualmente 100.416 casos confirmados da COVID-19, estando na segunda colocação dentre as regiões do país, atrás apenas da Sudeste, com 113.609 casos notificados. Destaca-se que os estados do Ceará, Pernambuco, Maranhão e Bahia, situados nesta região, estão entre os dez que mais acumulam casos, sobressaindo-se Ceará e Pernambuco entre os cinco com maior número de óbitos ${ }^{18}$.

Os documentos analisados foram obtidos por meio de acesso à página do Ministério da Saúde dedicada à COVID-19, onde estão armazenados para consulta pública os planos de contingência e outros documentos normativos elaborados pelo Governo Federal, bem como pelos governantes de todos os estados brasileiros ${ }^{18}$. A busca foi realizada no dia 5 de maio de 2020, a partir da qual os documentos acessados e selecionados foram armazenados para posterior análise.

O processo de análise considerou a identificação dos seguintes aspectos componentes dos planos: (1) organização e estrutura formal; (2) objetivos definidos, gerais e específicos; e (3) propostas definidas para a Vigilância em Saúde em âmbito estadual.

Para sua operacionalização, foram construídos quadros de análise preenchidos individualmente, contemplando tais aspectos em cada um dos planos considerados. Foi desenvolvida a análise de conteúdo do material produzido pelos estados, cujos achados foram refletidos tomando por referência o modelo político-operacional da Vigilância em Saúde ${ }^{19}$.

\section{RESULTADOS E DISCUSSÃO}

Todos os estados nordestinos construíram planos de contingência face à pandemia da COVID-19, seguindo as recomendações do Governo Federal ${ }^{5,20}$. Os estados de Alagoas e Paraíba publicaram os seus planos ainda em janeiro de 2020, antevendo a chegada do agravo no território nacional. Os planos da Bahia e de Pernambuco datam do mês de fevereiro e os do Rio Grande do Norte, de abril de 2020. Os demais estados não faziam menção ao mês de publicação.

De modo geral, há uma heterogeneidade na organização dos documentos, bem como nas ações programáticas e estratégicas a serem desenvolvidas no contexto da pandemia. No âmbito das ações de Vigilância em Saúde, a articulação dos estados com os seus respectivos municípios é referida nos planos de sete dos nove estados do Nordeste. As exceções são os estados do Maranhão e Paraíba.

O Quadro 1 sintetiza os planos estaduais analisados e as articulações previstas, no âmbito da Vigilância em Saúde, a serem desenvolvidas (ou não) junto aos municípios 21,22,23,24,25,26,27,28,29.

As ações das Vigilâncias em Saúde estaduais previstas na articulação com os municípios vão do suporte para o monitoramento e acompanhamento de casos (planos de Alagoas, Bahia, Ceará, Piauí e Sergipe 21,22,23,27,29) à pactuação nas Comissões Intergestores Bipartite e Comissão Intergestores Regionais (plano de Pernambuco $\left.{ }^{26}\right)$, bem como o suporte para que os municípios elaborem

Quadro 1. Caracterização dos planos de contingência dos estados da Região Nordeste do Brasil, quanto à previsão de articulações intra e intersetoriais da Vigilância em Saúde.

\begin{tabular}{|lcccc|}
\hline UF & Data de publicação & Data de atualização & $\begin{array}{c}\text { Articulação da Vigilância em Saúde } \\
\text { estadual com os municípios }\end{array}$ & $\begin{array}{c}\text { Articulações intra e intersetoriais } \\
\text { da Vigilância em Saúde estadual* }\end{array}$ \\
\hline AL & $30 / 01 / 2020$ & $1^{\text {a }} 08 / 02 / 2020$ & Prevê & Prevê \\
BA & Fevereiro/2020 & $2^{\mathrm{a}} 12 / 03 / 2020$ & Prevê & Prevê \\
CE & $2020^{*}$ & Não cita & Prevê & Não cita \\
MA & $2020^{*}$ & Não cita & Não cita & Prevê \\
PB & Janeiro/2020 & Não cita & Não cita & Prevê \\
PE & Fevereiro/2020 & Não cita & Prevê & Não cita \\
PI & $2020^{*}$ & Não há & Prevê & Prevê \\
RN & $02 / 04 / 2020$ & Não cita & Prevê & Não cita \\
SE & $2020^{*}$ & Versão preliminar & Prevê & Prevê \\
\hline
\end{tabular}

Fonte: Elaborado pelos autores, 2020.

UF: unidade federativa; AL: Alagoas; BA: Bahia; CE: Ceará; MA: Maranhão; PB: Paraíba; PE: Pernambuco; PI: Piauí; RN: Rio Grande do Norte; SE: Sergipe. * Cita apenas o ano. 
os seus planos de contingência ao enfrentamento da COVID-19 (plano do Rio Grande do Norte ${ }^{28}$ ).

O plano do Maranhão ${ }^{24}$, embora mencione as unidades básicas de saúde, que são de responsabilidade municipal, se restringe apenas à notificação de casos por esses estabelecimentos de saúde, enquanto o plano da Paraíba ${ }^{25}$ não aborda nenhuma articulação direta da Vigilância em Saúde Estadual com os municípios.

No tocante às articulações das Vigilâncias em Saúde estaduais com outros setores, permanece a heterogeneidade das ações, sendo planejadas: a atuação conjunta com os diferentes níveis de assistência à saúde; a interface com órgão do governo de abrangência nacional e a intersetorialidade com movimentos sociais, com as áreas da educação, justiça e demais setores economicamente produtivos. 0 Quadro 2 descreve as ações previstas.

Vale ressaltar que em três dos nove estados do Nordeste, nos seus respectivos planos, não estão descritas atividades articuladas da Vigilância em Saúde com outros órgãos do setor saúde, nem mesmo com instituições relacionadas a outros setores. Quanto aos demais, apesar de sinalizarem ações integradas da vigilância com outros serviços envolvidos no enfrentamento à COVID-19, apenas dois estados explicitam em seus planos, a articulação com outros setores, públicos e/ou privados, como educação, justiça, entidades sindicais e comerciais, entre outros, a exemplo das instituições de ensino e o Ministério Público (no plano da Bahia ${ }^{22}$ ), e os sindicatos de trabalhadores e os setores hoteleiro e alimentício (no plano do Piauí27).

Em linhas gerais, os planos apresentam possíveis caminhos e estratégias para o enfrentamento da pandemia, levando em consideração o nível de institucionalização da Vigilância em Saúde, no âmbito da gestão das práticas e ações em saúde, bem como visibilizam os recursos e atores disponíveis nos territórios dos estados para a produção de respostas em uma situação pandêmica.

Destacamos, neste sentido, a existência de uma diversidade discursiva e não discursiva que circula a partir destes materiais. Os quais são importantes instrumentos de governo, produtos e produtores de práticas sociais. Constituem objetos dotados de capacidade de ação e é neste sentido que são aqui tomados, pois agenciam diferentes práticas e produzem efeitos a partir das relações que estabelecem.

Neste sentido, em sua análise foi possível considerar a forma como as equipes gestoras envolvidas na elaboração dos respectivos planos situam o papel da Vigilância em Saúde em nível estadual, ainda que não se possa revelar como tais construções resultam efetivamente em seu processo de desenvolvimento e aplicação quanto às ações planejadas, mesmo reconhecendo sua capacidade de orientar as práticas de saúde.

A análise de tais documentos possibilitou a identificação de uma série de elementos que remontam aos processos de âmbito gerencial e da interface com outros setores. Como resultantes desse exercício analítico, foram denotadas duas categorias temáticas principais: 1) Aspectos políticos-gerenciais da Vigilância em Saúde em âmbito estadual e 2) Articulações organizacionais da Vigilância em Saúde.

Aspectos políticos-gerenciais da Vigilância em Saúde em âmbito estadual

A implantação do SUS, orientado pelo princípio constitucional do direito universal à saúde por toda a população brasileira, sendo uma

Quadro 2. Descrição das articulações intra e intersetoriais previstas nas ações das Vigilâncias em Saúde dos estados da Região Nordeste do Brasil.

\begin{tabular}{|c|c|}
\hline UF & Ações integradas \\
\hline $\mathrm{AL}$ & $\begin{array}{l}\text { Promover ações integradas entre Vigilância em Saúde, assistência, Anvisa, e outros órgãos envolvidos na prevenção e controle do novo } \\
\text { coronavírus (2019-nCoV). }\end{array}$ \\
\hline BA & $\begin{array}{l}\text { Articulação e realização de reuniões com outros setores que têm interface com as ações de enfrentamento do vírus } 2019 \text {-nCoV e outros vírus } \\
\text { Influenza de interesse de saúde pública: COSEMS, Anvisa, estabelecimentos de ensino, Conselhos de Saúde, Ministério Público, dentre outros. } \\
\text { Articulação e realização de reuniões com Conselhos de Saúde e Sociedade Civil organizada para envolvimento nas ações de prevenção e } \\
\text { controle do vírus } 2019-n C o V \text { e outros vírus respiratórios de interesse de saúde pública. }\end{array}$ \\
\hline CE & Não cita. \\
\hline MA & Apenas a notificação dos casos acolhidos nas Unidades Básicas de Saúde, em até 24 h, ao CIEVS estadual. \\
\hline PB & $\begin{array}{l}\text { A presença da Vigilância Epidemiológica no âmbito da Atenção Primária em Saúde e da Atenção Hospitalar será essencial para identificação } \\
\text { de possível caso suspeito. }\end{array}$ \\
\hline PE & Não cita. \\
\hline $\mathrm{PI}$ & $\begin{array}{l}\text { Articular reuniões, em parceria com as Visa municipais, com o setor regulado (representantes do comércio varejista e atacadista, sindicato } \\
\text { dos trabalhadores, clínicas, hospitais, drogarias, farmácias de manipulação, laboratórios, serviços de hotelaria e alimentação em geral, } \\
\text { serviço de transporte rodoviário, escola de todos os níveis, entre outros), com o objetivo de orientar sobre as medidas preventivas em } \\
\text { relação à redução do risco de contaminação do } 2019 \text {-nCoV. } \\
\text { Realizar busca ativa de todas as pessoas que tiveram ou têm contato com pessoas suspeitas ou confirmadas de COVID-19 e, juntamente com } \\
\text { a equipe da Visa, notificar os sintomáticos respiratórios e realizar ações de cuidado para o encaminhamento pela rede; da Atenção Primária } \\
\text { à Saúde com a Visa. }\end{array}$ \\
\hline RN & Não cita. \\
\hline SE & $\begin{array}{l}\text { Promover capacitação através do Telessaúde com os profissionais de saúde da Atenção Primária à Saúde, média e alta complexidade e } \\
\text { Vigilâncias em Saúde de todo estado. }\end{array}$ \\
\hline
\end{tabular}

Fonte: Elaborado pelos autores, 2020.

UF: unidade federativa; AL: Alagoas; BA: Bahia; CE: Ceará; MA: Maranhão; PB: Paraíba; PE: Pernambuco; PI: Piauí; RN: Rio Grande do Norte; SE: Sergipe; Anvisa: Agência Nacional de Vigilância Sanitária; COSEMS: Conselho das Secretarias Municipais de Saúde; CIEVS: Centro de Informações Estratégicas e Resposta de Vigilância em Saúde; Visa: Vigilância Sanitária. 
responsabilidade do Estado a sua garantia, implicou uma organização dos serviços, sob o comando único em cada esfera de governo ${ }^{30}$. A descentralização do SUS, proposta pela Constituição Federal de 1988, conformou que a gestão do sistema fosse compartilhada entre a União, os estados e os municípios, respeitando a autonomia de cada ente federado, com as decisões baseadas em consenso, assegurando a participação da sociedade (controle social) ${ }^{31}$.

A descentralização, como um dos princípios organizativos do SUS, acumula avanços significativos, como a redistribuição das responsabilidades e descentralização dos recursos, no âmbito da saúde. Esse gerenciamento local das políticas de saúde, por meio do compartilhamento do poder decisório, das instâncias de pactuação e novos instrumentos de participação social, objetiva a prestação de serviços com mais eficiência e qualidade ${ }^{30,32}$.

Um dos principais avanços desse processo de descentralização do setor saúde, ocorrido desde os anos de 1990, foi a assunção dos municípios como principais responsáveis pela gestão da rede de serviços de saúde no país, tendo a gestão municipal um papel fundamental para a execução de ações e serviços de saúde com o desafio de atender às particularidades da população de cada município.

Dessa forma, uma boa gestão da Vigilância em Saúde requer uma relação federativa cooperada entre os estados e seus respectivos municípios ${ }^{30,33}$. Os planos estaduais materializam a construção de uma agenda estratégica, na perspectiva de qualificar as ações para uma resposta efetiva face à pandemia da COVID-19. Esses documentos articulam decisões político-institucionais e o estabelecimento de mecanismos técnicos e dispositivos organizacionais, prevendo, inclusive, a divulgação de informações estratégicas relativas à pandemia.

A necessidade de novos arranjos institucionais no âmbito da Vigilância em Saúde demanda a promoção de ações descentralizadas e integradas, bem como articulações intersetoriais, que diminuam a fragmentação do trabalho e fortaleçam a parceria entre os estados e municípios. Neste caso, chama a atenção que dentre os nove planos analisados, os estados do Maranhão e da Paraíba, não mencionem pactuações com os municípios.

No sistema de legislação da saúde, a Portaria n 399, de 22 de fevereiro de 2006, do Ministério da Saúde, institui o pacto de gestão do SUS, estabelecendo responsabilidades claras de cada ente federado, de forma a diminuir as competências concorrentes, e a tornar mais claro quem deve fazer o quê, contribuindo, assim, para o fortalecimento da gestão compartilhada e solidária do SUS $^{34}$.

Segundo Miranda ${ }^{35}$, a gestão governamental em saúde se constitui por meio de uma práxis social de âmbito institucional, possuindo uma natureza complexa e polivalente. Na sua operacionalização está conformada uma dimensão política, a partir da intermediação de interesses distintos e das interações de poder e margens de autonomia, expressos em diferentes racionalidades de ação. 0 propósito primordial da gestão em saúde é a produção de decisões, sendo indispensável o exercício constante de relações intergovernamentais com o objetivo de ampliar o potencial de consenso e que desencadeiem implementações de políticas ${ }^{35}$.
Neste sentido, entendemos que, no contexto da pandemia, caso haja algum desequilíbrio nas relações entre os entes federados, via fragilização na pactuação intergovernamental, pode ser diminuída a capacidade de respostas dos sistemas locais de saúde, bem como haver a descontinuidade das ações de vigilância, resultando, por exemplo: no enfraquecimento dos sistemas de monitoramento e notificação e na fragmentação dos fluxos assistenciais que demandam da atuação da Vigilância em Saúde, tendo em vista a comunicação entre as redes de saúde municipais (que identificam casos, via atenção primária) e os leitos hospitalares, sob gestão dos estados.

\section{Articulações organizacionais da Vigilância em Saúde}

Outro ponto destacado na análise dos planos elaborados pelos estados do Nordeste para o enfrentamento da COVID-19 foi a articulação da Vigilância em Saúde com outros serviços e programas componentes da rede de saúde, bem como aqueles referentes a setores externos à saúde, como os movimentos sociais.

As ações previstas nos planos envolvem desde o apoio na identificação de casos suspeitos - como consta no plano do Piauí27 - a "articulação e realização de reuniões com Conselhos de Saúde e Sociedade civil organizada para envolvimento nas ações de prevenção e controle do vírus 2019-nCoV e outros vírus respiratórios de interesse de saúde pública", conforme apontado no plano do estado da Bahia ${ }^{22}$.

Exemplos como esse apontam para mudanças no modelo de organização dos serviços de saúde, visto que a Vigilância em Saúde ainda se ancora no modelo ecológico da doença e na epidemiologia clássica, tendo como objeto o controle dos modos de transmissão dos agravos e os fatores de risco/vulnerabilizantes ${ }^{30,32,36}$. Compreensão que possibilita uma governabilidade e eficácia das ações voltadas às doenças infectocontagiosas clássicas. Nessa proposta, a Vigilância em Saúde inclui o monitoramento do ambiente e de possíveis casos que atuam como eventos sentinelas, em associação com análises epidemiológicas ${ }^{30}$.

No contexto da pandemia da COVID-19, a maior parte das respostas inscritas nos planos estaduais tem por base um outro processo saúde-doença. $\mathrm{Na}$ interface com as contribuições das ciências sociais e humanas na saúde, visibilizam os processos sociais que promovem ou agravam a saúde das populações. Nessa perspectiva, os limites das ações de Vigilância em Saúde esbarram em características da própria sociedade, a exemplo do nível de equidade, dos processos de participação social na elaboração das estratégias de atuação $0^{30,32,33}$.

Contudo, convém destacar que essas práticas clássicas de vigilância coexistem nos sistemas de saúde e se fazem presente nos planos estaduais analisados, inclusive com uma setorização da Vigilância em Saúde. A Vigilância Epidemiológica é responsável pelo controle dos agravos, a definição de casos suspeitos e a implantação de medidas emergenciais de isolamento dos pacientes. A Vigilância Sanitária atua como responsável pelo suporte aos serviços de saúde e dos fármacos. E a "vigilância laboratorial", na realização dos exames dos casos suspeitos de COVID-19, como previsto nos planos de contingência dos estados 
de Alagoas, Pernambuco e Paraíba, sugerindo uma nova subdivisão da Vigilância em Saúde $21,22,23,24,25,26,27,28,29$.

Essa setorização aponta para alguns problemas centrais da Vigilância em Saúde no desenvolvimento de ações de prevenção e promoção. Na análise dos planos, verifica-se a inexistência de um delineamento claro dos papeis e limites das ações de âmbito intrassetoriais da saúde, e/ou nas ações em parceria com outros setores.

A lógica clássica de intervenção sanitária, não consegue responder aos problemas nos sistemas de saúde no ensejo da pandemia. Quanto mais distantes da perspectiva ampliada de Vigilância em Saúde, maior a necessidade da construção de práticas alternativas que superem os limites deste tipo de compreensão $0^{30,31,36}$.

Nesse contexto sanitário, uma revisão epistemológica é importante. Ou seja, verificar os atuais reducionismos e possibilidades de avanços conceituais e metodológicos perante esse processo saúde-doença pandêmico. No âmbito político-institucional, significa avançar em práticas intersetoriais e na relação com a sociedade. 0 entendimento mais ampliado e significativo dos determinantes e condicionantes da saúde é a principal base para a construção de novas práticas de Vigilância em Saúde.

\section{CONCLUSÕES}

A pandemia da COVID-19 trouxe uma série de desafios ao SUS na produção de respostas articuladas e efetivas para a proteção da população brasileira. Neste âmbito, os aspectos político-institucionais e de articulação da Vigilância em Saúde são fundamentais.

A atuação integrada e compartilhada da Vigilância em Saúde nos diversos níveis de governo está prevista no arcabouço normativo do SUS. No contexto nordestino, além da heterogeneidade dos municípios, há que considerar ainda fragilidades institucionais na gestão local do sistema de saúde, visto que a região Nordeste concentra $63,4 \%$ de municípios do grupo de pequeno porte do país ${ }^{37}$. Características que apontam a necessidade de uma atuação coordenada por parte do nível estadual para a elaboração de respostas mais efetivas.

A pandemia da COVID-19, além dos altos índices de transmissibilidade e mortalidade, também vem visibilizando a fragilidade institucional da gestão do SUS. Assim, em um cenário pandêmico, o planejamento das ações possui um fluxo não habitual e mais acelerado face à gravidade da situação sanitária e do perfil epidemiológico da doença. Ante este cenário é que a atuação da Vigilância em Saúde deve ir além do levantamento de dados, fomentando a tomada de decisão e pautando o planejamento e execução das ações.

Em locais com capacidade organizativo-institucional limitada, a produção de respostas requer amplo suporte e, no caso em questão, ofertado pelos governos estaduais. Na análise realizada, destacam-se os planos estaduais do Maranhão e da Paraíba, os quais, no âmbito da Vigilância em Saúde, não preveem articulações com as vigilâncias municipais, seja para elaborar seus respectivos planos de enfrentamento, seja para desenvolver atividades conjuntas. Podemos refletir que a publicação prematura de tais planos, considerando que este último (Paraíba) foi disponibilizado antes mesmo de a COVID-19 ser confirmada em território nacional, pode sinalizar uma carência no amadurecimento das ações propostas, bem como no seu próprio processo de elaboração.

A respeito da articulação intrassetorial da Vigilância em Saúde é necessária a interface com os demais serviços e programas de saúde locais e regionais; bem como o compartilhamento com outras áreas além do âmbito da saúde, incluindo outros atores importantes para a construção de intervenções integradas com vistas a potencializar os efeitos benéficos e de proteção da população.

Não obstante, é importante considerar o alcance das análises desenvolvidas neste artigo, uma vez que pretensamente se buscou lançar luz sobre a forma como a Vigilância em Saúde é inscrita em documentos normativos que guiam a condução de políticas via produção discursiva das equipes da gestão estadual. Os planos de contingência constituem documentos que visam guiar a ação pública desenvolvida pelos entes da gestão, e como tal são potenciais produtores de sentidos sobre a forma como as ações são pensadas e serão operacionalizadas no âmbito da política de saúde.

No entanto, há que se destacar as limitações do escopo das problematizações aqui realizadas, tendo em vista a impossibilidade de ponderar as aplicabilidades práticas dos referidos atos normativos, tanto pela forma como tais documentos foram construídos, contando com maior ou menor participação dos atores responsáveis pela implementação das ações, quanto pelas rápidas transformações que ocorrem na organização dos sistemas de saúde locais, tendo em vista as incertezas que acompanham a evolução da pandemia da COVID-19 no Brasil.

\section{REFERÊNCIAS}

1. World Health Organization - WHO. WHO director-general's opening remarks at the media briefing on COVID-19: 11 march 2020. WHO Speeches. 11 mar 2020[acesso $7 \mathrm{abr}$ 2020]. Disponível em: https://www.who.int/dg/speeches/ detail/who- director-general-s-opening-remarks-at the-media-briefing-on-COVID-19-11-march-2020

2. Sarti TD, Lazarini WS, Fontenelle LF, Almeida APSC. Qual o papel da atenção primária à saúde diante da pandemia provocada pela COVID-19? Epidemiol Serv Saude. 2020;29(2):1-5. https://doi.org/10.5123/s1679-49742020000200024
3. World Health Organization - WHO. Statement on the second meeting of the international health regulations (2005) emergency committee regarding the outbreak of novel coronavirus (2019-nCoV). WHO Newsroom. 30 jan 2020[acesso 7 abr 2020]. Disponível em: https: //www.who.int/news-room/ detail/30-01-2020-statement-on-the-second-meetingof-the-international-health-regulations-(2005)emergency-committee-regarding-the-outbreak-of-novelcoronavirus-(2019-ncov) 
4. World Health Organization - WHO. Coronavirus disease (COVID-19) outbreak. WHO Emergencies Diseases. 2020[acesso 13 maio 2020]. Disponível em: https://www.who.int/emergencies/diseases/ novel-coronavirus-2019

5. Ministério da Saúde (BR). Portaria $N^{\circ} 188$, de 3 de fevereiro de 2020. Declara emergência em saúde pública de importância nacional (Espin) em decorrência da infecção humana pelo novo coronavírus (2019-nCoV). Diário Oficial União. 4 fev 2020.

6. Garcia LP, Duarte E. Intervenções não farmacológicas para o enfrentamento à epidemia da COVID-19 no Brasil. Epidemiol Serv Saude. 2020;29(2):1-4. https://doi.org/10.5123/s1679-49742020000200009

7. Barreto ML, Barros AJD, Carvalho MS, Codeço CT, Hallal PRC, Medronho RA et al. $O$ que é urgente e necessário para subsidiar as políticas de enfrentamento da pandemia de COVID-19 no Brasil? Rev Bras Epidemiol. 2020;23:1-4. https://doi.org/10.1590/1980-549720200032

8. Corrêa Filho HR, Segall-Corrêa AM. Lockdown ou vigilância participativa em saúde? Lições da COVID-19. Saude Debate. 2020;44(124):5-10. https://doi.org/10.1590/0103-1104202012400.

9. Diretoria Nacional. A gestão municipal e a garantia do direito à saúde. Saude Debate. 2012;36(95):506-11. https://doi.org/10.1590/S0103-11042012000400001

10. Senado Federal (BR). Constituição da República Federativa do Brasil. Brasília: Senado Federal; 1988.

11. Ministério da Saúde (BR). Coordenação-geral e apoio à gestão descentralizada, departamento de apoio à descentralização, secretaria executiva, ministério da saúde: diretrizes operacionais dos pactos pela vida, em defesa do SUS e de gestão. Brasília: Ministério da Saúde; 2006.

12. Teixeira CF, Molesini JA. Gestão municipal do SUS: atribuições e responsabilidades do gestor do sistema e dos gerentes de unidades de saúde. Rev Baiana Saude Publica. 2002;26(1-2):29-40. https://doi.org/10.22278/2318-2660.2002.v26.n1-2.a983

13. Ministério da Saúde (BR). Portaria $N^{\circ} 1.378$, de 9 de julho de 2013. Regulamenta as responsabilidades e define diretrizes para execução e financiamento das ações de vigilância em saúde pela união, estados, Distrito Federal e municípios, relativos ao Sistema Nacional de Vigilância em Saúde e Sistema Nacional de Vigilância Sanitária. Diário Oficial União. 10 jul 2013.

14. Ministério da Saúde (BR). Guia de vigilância epidemiológica. 6a ed. Brasília: Ministério da Saúde; 2005.

15. Spink PK. Análise de documentos de domínio público. In: Spink MJP, organizadora. Práticas discursivas e produção de sentidos no cotidiano: aproximações teóricas e metodológicas. São Paulo: Cortez; 2000. p. 123-51.

16. Spink PK. O pesquisador conversador no cotidiano. Psicol Soc. 2008;20(esp):70-7. https://doi.org/10.1590/\$0102-71822008000400010
17. Spink PK. Pesquisa de campo em psicologia social: uma perspectiva pós-construcionista. Psicol Soc. 2003;15 (2):8-42. https://doi.org/10.1590/S0102-71822003000200003

18. Ministério da Saúde (BR). Painel coronavírus. Coronavírus Brasil. 21 maio 2020[acesso 21 maio 2020]. Disponível em: https://covid.saude.gov.br/

19. Teixeira CF. Promoção e vigilância da saúde no contexto da regionalização da assistência à saúde no SUS. Cad Saude Publica. 2002;18(Suppl):S153-62. https://doi.org/10.1590/S0102-311X2002000700015

20. Ministério da Saúde (BR). Centro de operações de emergências em saúde pública COE-COVID-19: plano de contingência nacional para infecção humana pelo novo coronavírus COVID-19. Brasília: Ministério da Saúde; 2020[acesso 7 abr 2020]. Disponível em: https://portalarquivos2.saude.gov.br/images/pdf/2020/ fevereiro/13/plano-contingencia-coronavirus-COVID19.pdf

21. Secretaria de Estado da Saúde de Alagoas - SES-AL. Plano de contingência estadual para infecção humana pelo novo coronavírus 2019-nCoV. Maceió: Secretaria de Estado da Saúde de Alagoas; 2020.

22. Secretaria de Saúde do Estado da Bahia - SSE-BA. Plano estadual de contingências para enfrentamentodo novo coronavírus: 2019-n CoV. Salvador: Secretaria de Saúde do Estado da Bahia; 2020.

23. Secretaria da Saúde do Estado do Ceará - SSE-CE. Plano estadual de contingência para resposta às emergências em saúde pública: novo coronavírus (2019-nCoV). Fortaleza: Secretaria da Saúde do Estado do Ceará; 2020.

24. Secretaria de Estado da Saúde do Maranhão - SES-MA. Plano estadual de contingência do novo coronavírus 2019-nCoV. São Luís: Secretaria de Estado da Saúde do Maranhão; 2020.

25. Secretaria de Estado da Saúde da Paraíba - SES-PA. Plano de contingência estadual para infecção humana pelo novo coronavírus (2019-nCoV). João Pessoa: Secretaria de Estado da Saúde da Paraíba; 2020.

26. Secretaria Estadual de Saúde de Pernambuco - SES-PE. Plano de contingência para infecção humana pelo novo coronavírus (2019-nCoV). Recife: Secretaria Estadual de Saúde de Pernambuco; 2020.

27. Secretaria de Estado da Saúde do Piauí - SES-PI. Plano estadual de contingência para o enfrentamento da infecção humana pelo coronavírus (2019- nCoV) do estado do Piauí. Teresina: Secretaria de Estado da Saúde do Piauí; 2020.

28. Secretaria de Estado da Saúde Pública do Rio Grande do Norte - SESP-RN. Plano de contingência estadual para infecção humana pelo COVID-19. Natal: Secretaria de Estado da Saúde Pública do Rio Grande do Norte.; 2020.

29. Secretaria de Estado da Saúde de Sergipe - SES-SE. Plano de contingência estadual para infecção humana pelo novo coronavírus 2019-nCoV. Aracajú: Secretaria de Estado da Saúde de Sergipe; 2020.

30. Oliveira CM, Cruz MM. Sistema de Vigilância em Saúde no Brasil: avanços e desafios. Saude Debate. 2015;39(104):255-67. https://doi.org/10.1590/0103-110420151040385 
31. Teixeira MG, Costa MCN, Carmo EH, Oliveira WK, Penna GO. Vigilância em Saúde no SUS: construção, efeitos e perspectivas. Cienc.Saude Coletiva. 2018 jun;23(6):1811-8. http://dx.doi.org/10.1590/1413-81232018236.09032018

32. Recktenwaldt $M$, Junges JR. A organização e a prática da Vigilância em Saúde em municípios de pequeno porte. Saude Soc. 2017;26(2):367-81. https://doi.org/10.1590/s0104-12902017171916

33. Medeiros EHF, Machado MF, Pessoa Júnior JM. Avaliação da organização estrutural das vigilâncias sanitárias municipais de uma região de saúde de Pernambuco. Physis. 2017;27(3):561-78. https://doi.org/10.1590/s0103-73312017000300010

34. Ministério da Saúde (BR). Portaria № 399, de 22 de fevereiro de 2006. Divulga o pacto pela saúde 2006: consolidação do SUS e aprova as diretrizes operacionais do referido pacto. Diário Oficial União. 23 fev 2006.

35. Miranda AS. Proposição de escopo para a avaliação da gestão no sistema único de saúde: relatório final de consultoria prestada ao ministério da saúde. Brasília: Ministério da Saúde; 2005.

36. Fernandes VR, Luz ZP, Amorim AC, Sérgio JV, Silva JPV, Castro MC et al. O lugar da vigilância no SUS: entre os saberes e as práticas de mobilização social. Cienc Saude Coletiva. 2017;22(10):3173-81. https://doi.org/10.1590/1413-812320172210.1772017

37. Calvo MCM, Lacerda JT, Colussi CF, Schneider IJC, Rocha TAH. Estratificação de municípios brasileiros para avaliação de desempenho em saúde. Epidemiol Serv Saude. 2016;25(4):767-76. https://doi.org/10.5123/s1679-49742016000400010

\section{Contribuição dos Autores}

Machado MF - Concepção, planejamento (desenho do estudo), aquisição, análise, interpretação dos dados e redação do trabalho. Quirino TRL - Planejamento (desenho do estudo), interpretação dos dados e redação do trabalho. Souza, CDF- Interpretação dos dados e redação do trabalho. Todos os autores aprovam a versão final do trabalho.

Conflito de Interesse

Os autores informam não haver qualquer potencial conflito de interesse com pares e instituições, políticos ou financeiros deste estudo.

Esta publicação está sob a licença Creative Commons Atribuição 3.0 não Adaptada.

Para ver uma cópia desta licença, visite http://creativecommons.org/licenses/by/3.0/deed.pt_BR. 